\title{
TOTALLY INTEGRALLY CLOSED RINGS
}

\section{EDGAR ENOCHS}

All rings and algebras in the following will be assumed commutative and associative. All rings will have identities, and ring homomorphisms will map the identity onto the identity. The identity of a subring will always be that of the ring.

We recall that if $A$ is a subring of a ring $B$, then $b \in B$ is said to be integral over $A$ if there is a unitary polynomial $f \in A[X]$ such that $f(b)=0 . B$ is said to be an integral extension of $A$ if each $b \in B$ is integral over $A$. If $B$ is an integral extension of $A$, then for every prime ideal $\odot$ of $A$ there is a prime ideal $Q$ of $B$ with $Q \cap A=\odot$. If $B$ is any extension of the ring $A$, the set $A^{\prime}$ of $b \in B$ which are integral over $A$ is a subring of $B$ and so an integral extension of $A$. If $A=A^{\prime}$, $A$ is said to be integrally closed in $B$. If $A \subset B \subset C$ where $C$ is a ring and $A$ and $B$ are subrings, then if $C$ is an integral extension of $B$ and $B$ an integral extension of $A$ then $C$ is an integral extension of $A$. From this it follows that the integral closure $A^{\prime}$ of $A$ in an extension $B$ is integrally closed in $B$.

In the category of fields, integral extensions are just the algebraic extensions. The algebraically closed fields $\Omega$ are precisely those which are injective with respect to algebraic extensions, i.e. they have the property that if $\sigma: K \rightarrow \Omega$ is a homomorphism where $K$ is a field and $E$ is an algebraic extension of $K$, then there is a homomorphism $E \rightarrow \Omega$ agreeing with $\sigma$ on $K$. Each field $K$ has an algebraic extension $\Omega$ which is algebraically closed. Furthermore, if $\Omega^{\prime}$ is another algebraically closed algebraic extension of $K$, then any $k$-homomorphism $\Omega \rightarrow \Omega^{\prime}$ (which always exists) is an isomorphism. The question naturally rises whether we get the analogous result in the category of rings when we consider integral extensions. In this paper we consider this problem.

Definition. $A$ ring $D$ is said to be totally integrally closed if for any ring homomorphism $\sigma: B \rightarrow D$ and any integral extension $C$ of $B$ there is a homomorphism $C \rightarrow D$ extending $\sigma$. (The term integrally closed is reserved for an integral domain which is integrally closed in its field of fractions.)

The following two propositions are immediate.

Proposition 1. If $\left(A_{i}\right)_{i \in I}$ is a family of rings, then $\prod_{i \in I} A_{i}$ is

Received by the editors March 13, 1967. 
totally integrally closed if and only if each ring $A_{i}$ is totally integrally closed.

Proposition 2. If $A$ is a subring of a ring $B$ and $A$ is a retract of $B$ (i.e. there is a homomorphism $r: B \rightarrow A$ with $r \mid A=1_{A}$ ), then if $B$ is totally integrally closed, $A$ is totally integrally closed.

The following proposition gives examples of totally integrally closed rings.

Proposition 3. An integral domain $A$ is totally integrally closed if and only if $A$ is integrally closed in an algebraic closure $\Omega$ of its field of fractions $K$.

Proof. Suppose $A$ is integrally closed in $\Omega$ and let $\sigma: B \rightarrow A$ be a ring homomorphism and let $C$ be an integral extension of $B$. Then $\operatorname{Ker}(\sigma)$ is a prime ideal of $B$ since $A$ is an integral domain. Since $C$ is an integral extension of $B$ there is a prime ideal $P$ of $C$ such that $\odot \cap B=\operatorname{Ker}(\sigma)$. Let $B^{\prime}=B / \operatorname{Ker}(\sigma)$ and $C^{\prime}=C / \odot$. We consider $B^{\prime}$ a subring of $C^{\prime}$. We have an induced injective homomorphism $B^{\prime} \rightarrow A$, and so a homomorphism $B^{\prime} \rightarrow \Omega$. Now let $M$ be a field of fractions of $C^{\prime}$ and $L \subset M$ be a field of fractions of $B^{\prime}$. Then $M$ is an algebraic extension of $L$ since $C$ is an integral extension of $B$. Now the injective homomorphism $B^{\prime} \rightarrow \Omega$ can be extended to $L \rightarrow \Omega$ and this in turn to a homomorphism $M \rightarrow \Omega$ since $\Omega$ is algebraically closed. Thus by restriction we get a homomorphism $C / \beta=C^{\prime} \rightarrow \Omega$ and so a homomorphism $\tau: C \rightarrow \Omega$ which agrees with $\sigma$ on $B$. Since $C$ is an integral extension of $B$, the elements of $\tau(C)$ are integral over $\tau(B)=\sigma(B) \subset A$ and hence belong to $A$ since $A$ is integrally closed in $\Omega$. Thus $\tau(C) \subset A$, and so $\sigma: B \rightarrow A$ has an extension $C \rightarrow A$.

Conversely, if $A$ is totally integrally closed, then by Proposition 2 above there is a retraction $r: B \rightarrow A$ where $B$ is the integral closure of $A$ in $\Omega$. Then $\operatorname{Ker}(r) \cap A=0$. But by the usual argument this is possible only if $\operatorname{Ker}(r)=0$, for if $b \in B, b \neq 0$, then suppose $b^{n}+a_{n-1} b^{n-1}$ $+\cdots+a_{1} b+a_{0}=0$ with $a_{i} \in A, i=0, \cdots, n-1$ where $n \geqq 1$ is minimal. Then $a_{0} \neq 0$ and $a_{0} \in \operatorname{Ker}(r)$. But $\operatorname{Ker}(r) \cap A=0$. Thus we see that $\operatorname{Ker}(r)=0$ and so $r$ is an isomorphism which implies $B=A$.

Now we have

Theorem 1. A ring $A$ is a subring of a totally integrally closed ring if and only if $A$ is reduced.

Proof. We first recall the well-known [1, Theorem 2.1, p. 4].

Lemma. If $A$ is a ring and $S \subset A$ is closed under multiplication and 
$0 \notin S$, then if $S \neq \varnothing$, any ideal of $A$ which is a maximal element in the set of ideals a such that $a \cap S=\varnothing$ is a prime ideal.

Now let $A$ be a reduced ring. If $x \in A, x \neq 0$, let $S$ be the set of $x^{n}$ for $n \in N$. Then by the lemma there is a prime ideal $\rho$ with $x \notin \rho$. Thus if $\operatorname{Spec}(A)$ is the set of prime ideals of $A$, we see we have an injective homomorphism $A \rightarrow \prod A / \odot$ where $\odot$ ranges through $\operatorname{Spec}(A)$. But each $A / \odot$ is an integral domain, so by Proposition 3

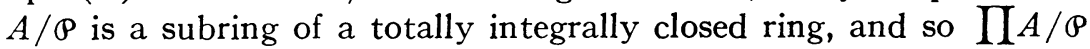
by Proposition 1 is a subring of a totally integrally closed ring. But then $A$ is a subring of a totally integrally closed ring.

Now suppose $A$ is not reduced. Then clearly there is a $c \in A$ with $c^{2}=0, c \neq 0$. Let $B$ be the set of $b \in A$ with $b c=0$. Let $A^{\prime}=A / B$. Now let $I$ be any nonempty set and let $\mathcal{F}(I)$ be the set of nonempty finite subsets of $I$. We consider the algebra $E$ over $A^{\prime}$ generated by elements $X, Y_{\imath}$ for $\iota \in I$, and $Z_{\nu}$ for $\nu \in \mathcal{F}(I)$ with the relations:

$X^{2}=Y_{\iota}^{2}=X Y_{\iota}=X Z_{\nu}=0$ for all $\iota \in I, \nu \in \mathcal{F}(I)$.

$Y Z_{\nu}=0$ if $\iota \notin \nu$ for all $\iota \in I, \nu \in \mathcal{F}(I)$.

$Z_{\nu}^{2}=Z_{\nu}$ for all $\nu \in \mathcal{F}(I)$.

$Z_{\nu} Y_{\iota_{1}} Y_{\iota_{2}} \cdots Y_{\iota_{n}}=X$ if $\nu \in \mathcal{F}(I), \nu=\left\{\iota_{1}, \cdots, \iota_{n}\right\}$, where $\iota_{i} \neq \iota_{j}$ if $i \neq j$.

Then we claim that every ideal of $E$ different from 0 contains a nonzero element of $A^{\prime} \cdot X$ and that $\operatorname{Card}(E) \geqq \operatorname{Card}(I)$.

For it's easy to see every element of $E$ can be written uniquely in the form

$$
a X+\sum_{a \iota_{1} \cdots \iota_{n}} Y_{\iota_{1}} \cdots Y_{\iota_{n}}+\sum_{b \iota_{1} \cdots \iota_{n}} Y_{\iota_{1}} \cdots Y_{\iota_{m}} Z_{v}
$$

where the first summations are over all sets $\left\{\iota_{1}, \cdots, \iota_{n}\right\}$ with $\iota_{i} \neq \iota_{j}$ if $i \neq j$, and the second over sets $\left\{\iota_{1}, \cdots, \iota_{m}\right\}$ and $\nu$ where $\left\{\iota_{1}, \cdots, \iota_{m}\right\}$ $\Phi \nu$ and $\iota_{i} \neq \iota_{j}$ if $i \neq j$. Now let $z \in E, z \neq 0$. If $z=a X$ for some $a \in A^{\prime}$, we are through. If not, by the relations above and the form in which we can write $z$, we see that for some $\nu \in \mathcal{F}(I), z Z_{\nu} \neq 0$, and so we can suppose that $z=\left(\sum b_{\iota_{i}} \cdots_{\iota_{m}} Y_{\iota_{j}} \cdots Y_{\iota_{m}}\right) Z_{\nu}$ where the summation is over $\left\{\iota_{i}, \cdots, \iota_{m}\right\} \subseteq \nu$ with $\iota_{i} \neq \iota_{j}$ if $i \neq j$. Then it's easy to see that multiplying $z$ by some of the $Y_{\imath}$ 's, we could assume $z \in A^{\prime} \cdot X$ and $z \neq 0$. Now clearly $\operatorname{Card}(E) \geqq \operatorname{Card}(I)$.

Now recalling that $A^{\prime}=A / B$, we make $E$ into an algebra over $A$ in the obvious fashion. Note then that $b z=0$ if $b \in \mathbb{B}, z \in E$. Now form the ring $A \times E$ with componentwise addition and multiplication

$$
(a, w) \cdot(b, z)=(a b, b w+a z+w z) .
$$

Then considering $A$ as a subring of $A \times E$, we see $A \times E$ is an inte- 
gral extension of $A$. Now let $\mathfrak{C}$ be the set of pairs $(a c, a X)$ for $a \in A$. This is clearly a subgroup of $A \times E$. If $b \in A, z \in E$, we have

$$
(b, o)(a c, a X)=(b a c, b a X), \quad(o, z)(a c, a X)=(o, a c z+a z X) .
$$

But $c a=0$ since $c \in B$ and $X z=0$. Thus we see $\mathcal{C}$ is an ideal of $A \times E$. Note that if $a c=0$ for some $a \in A$, then $a \in \mathbb{B}$ so $a X=0$. Thus $\mathfrak{e} \cap A$ $=0$. Now let $\bar{A}=A \times E / \mathrm{C}$. We identify $A$ with a subring of $\bar{A}$, so now $\bar{A}$ is an integral extension of $A$. Note we have $\operatorname{Card}(\bar{A}) \geqq \operatorname{Card}(I)$. Now let $D$ be an ideal of $\bar{A}$ such that $A \cap D=0$. Then $D=\mathfrak{C}^{\prime} / \mathfrak{C}$ where $\mathfrak{e}^{\prime} \supset \mathfrak{C}$ is an ideal of $A \times E$. If $(a, z) \in \mathfrak{C}^{\prime}$, then $a=0$ implies $z=0$, for otherwise, by the above, the subalgebra of $E$ (as an algebra over $A$ ) generated by $z$ would contain an element $a X, a \in A, a \notin B$. Thus $(0, a X) \in \mathfrak{e}^{\prime}$ but $\mathfrak{e}^{\prime} \supset \mathfrak{e}$, so $(-a c, 0) \in \mathfrak{e}^{\prime}$. But $a \notin \Theta$, so $a c \neq 0$ so that $A \cap D \neq 0$, which is a contradiction. Also if $(a, z) \in \mathbb{D}$ and $z=0$, we have $a=0$ since $A \cap D=0$. Thus we see that $\operatorname{Card}(D) \leqq C a r d(I)$.

Now suppose $A$ is a subring of a totally integrally closed ring $D$. Then there is a ring homomorphism $\sigma: \bar{A} \rightarrow D$ with $\sigma \mid A$ the canonical injection. Thus $\operatorname{Ker}(\sigma) \cap A=0$. By the above, this implies $\operatorname{Card}(\operatorname{Ker}(\sigma)) \leqq \operatorname{Card}(A)$. But $\operatorname{Card}(\bar{A}) \geqq \operatorname{Card}(I)$. Hence if we choose $I$ infinite and such that $\operatorname{Card}(A)>\operatorname{Card}(I)$, we get $\operatorname{Card}(\bar{A} / \operatorname{Ker}(\sigma))$ $=\operatorname{Card}(\bar{A})$. But then $\operatorname{Card}(D) \geqq \operatorname{Card}(I)$ for any such $I$, which is clearly impossible. This completes the proof of Theorem 1.

REMARK. Note that if a ring $A$ is a subring of a totally integrally closed ring $D$, then the integral closure $C$ of $A$ in $D$ is easily seen to be totally integrally closed and is an integral extension of $A$. However, the fact that two such extensions of $A$ are not necessarily isomorphic follows from the next proposition.

Proposition 4. If $A \neq 0$ is a reduced ring and $N_{\gamma}$ a cardinal number, there exists a totally integrally closed integral extension $D$ of $A$ with $\operatorname{Card}(D) \geqq N_{\gamma}$.

Proof. Let $B$ be a reduced integral extension of $A$. Then $B \times B$ with componentwise addition and multiplication

$$
(b, c)\left(b^{\prime}, c^{\prime}\right)=\left(b b^{\prime}, b c^{\prime}+c b^{\prime}+c c^{\prime}\right)
$$

is easily seen to be proper integral extension of $B$ and to be reduced. Thus we see that if $\beta$ is an ordinal number, we can construct totally integrally closed integral extensions $D_{\alpha}$ of $A$ for any $\alpha \leqq \beta$ such that if $\alpha<\alpha^{\prime} \leqq \beta, D_{\alpha}$ is a subring of $D_{\alpha^{\prime}}$ and $D_{\alpha} \neq D_{\alpha^{\prime}}$. But then by choosing $\beta$ sufficiently large we have $\operatorname{Card}\left(D_{\beta}\right) \geqq N_{\gamma}$.

In order to regain uniqueness we make the following.

Definition. An extension $B$ of a ring $A$ is said to be a tight exten- 
sion of $A$ if $B \cap A=0$ for any ideal $B$ of $B$, then $B=0$.

Then in complete analogy with the notion of an injective envelope [2, pp. 75-78], we have

TheOREM 2. If $A$ is a reduced ring, there is a totally integrally closed integral extension $A^{\prime}$ of $A$ which is also a tight extension of $A$. If $A^{\prime \prime}$ is any other such extension of $A$, then any $A$-homomorphism $A^{\prime} \rightarrow A^{\prime \prime}$ is an isomorphism.

Proof. The argument, with very slight modification, is that in [2, pp. 75-78]. We repeat it here for completeness. We first note that if $B$ is a tight extension of $A$ and $C$ a tight extension of $B$, then $C$ is a tight extension of $A$. Then suppose that $A$ is a subring of $B, B$ is a limit ordinal number, and $\left(A_{\alpha}\right)$ is a family of subrings of $B$ indexed by all $\alpha<\beta$ such that:

$A_{0}=A$.

$A_{\alpha+1}$ is a tight extension of $A_{\alpha}$ for all $\alpha<\beta$.

$A_{\gamma}=\bigcup A_{\alpha}$ for $\alpha<\gamma$ whenever $\gamma<\beta$ is a limit ordinal.

Then it's easy to check that $\mathrm{U} A_{\alpha}$ for $\alpha<\beta$ is a tight extension of $A$. From this, it follows that we can find a tight extension $A^{\prime}$ of $A$ in $B$ such that if $A^{\prime \prime}$ is a tight extension of $A^{\prime}, A^{\prime \prime} \subset B$ then $A^{\prime}=A^{\prime \prime}$. Now suppose $A$ is a reduced ring, $B$ is a totally integrally closed ring containing $A$. By the Remark following Theorem 1 we can furthermore suppose $B$ is an integral extension of $A$. Then it suffices to show the $A^{\prime}$ gotten above is totally integrally closed. By Proposition 2 it suffices to show $A^{\prime}$ is a retract of $B$. We choose an ideal $B$ of $B$ such that $A^{\prime} \cap B=0$, and furthermore we suppose $B$ is maximal with this property. Then we can identify $A^{\prime}$ with a subring of $B / B$. Clearly, $B / B$ is an integral extension of $A^{\prime}$. By the maximality of $B$ it easily follows that $B / B$ is a tight extension of $A^{\prime}$. Now $B$ totally integrally closed implies we can find an $A^{\prime}$-homomorphism

$$
B / B \stackrel{\sigma}{\rightarrow} B
$$

Then the fact that $B / B$ is a tight extension of $A^{\prime}$ implies this is an injection since $\operatorname{Ker}(\sigma) \cap A^{\prime}=0$. But then $\sigma(B / B)$ is a tight extension of $\sigma\left(A^{\prime}\right)=A^{\prime}$. By the choice of $A^{\prime}$ this implies $\sigma(B / B)=A^{\prime}$ so we get $A^{\prime}=B / B$ since $\sigma$ is an injection. This means that $A^{\prime}+B=B$ and, since $A^{\prime} \cap B=0$, that $A^{\prime}$ is a retract of $B$. Thus by Proposition $1, A^{\prime}$ is totally integrally closed.

Now if $A^{\prime}$ and $A^{\prime \prime}$ are both totally integrally closed, integral extensions of $A$, let $\sigma: A^{\prime} \rightarrow A^{\prime \prime}$ be an $A$-homomorphism. $\sigma$ is an injection since $\operatorname{Ker}(\sigma) \cap A=0$. Thus $\sigma\left(A^{\prime}\right) \cong A^{\prime}$ and so is totally integrally closed. But then there is a $\sigma\left(A^{\prime}\right)$-homomorphism $r: A^{\prime \prime} \rightarrow \sigma\left(A^{\prime}\right)$. But 
$\operatorname{Ker}(r) \cap \sigma\left(A^{\prime}\right)=0$ so $\operatorname{Ker}(r) \cap A=0$ so $\operatorname{Ker}(r)=0$. But then clearly $\sigma\left(A^{\prime}\right)=A^{\prime \prime}$. This completes the proof.

Remark 1. If $A$ is a reduced ring and every unitary $f \in A[X]$ has a root in $A$, it doesn't imply that $A$ is totally integrally closed. For example, let $A \subset \Omega^{I}$ with $I$ infinite, where $\Omega$ is an algebraically closed field and $A$ consists of all $\left(x_{\iota}\right)$ such that for some $x \in \Omega, x_{\iota}=x$ for all but a finite number of $\iota \in I$. Then $f \in A[X]$ is such that $f \in \Omega_{\iota_{1}} \times \Omega_{\iota_{2}}$ $\times \cdots \times \Omega_{\iota_{n}} \times \Delta[X]$ where $\Delta$ is the diagonal of $\Omega^{I^{\prime}}$ with $I^{\prime}=I$ $-\left\{\iota_{1}, \cdots, \iota_{n}\right\}$. But $\Delta=\Omega$ so clearly $f$ has a root in $\Omega_{\iota_{1}} \times \cdots \times \Omega_{\iota_{n}} \times \Delta$ and so is in $A$. But the integral closure $A^{\prime}$ of $A$ in $\Omega^{I}$ consists of all $\left(x_{\imath}\right)$ for which there is an $f \in \Omega[X], f \neq 0$ with $f\left(x_{\iota}\right)=0$ for all $\iota \in I$. There is such an $\left(x_{\imath}\right) \notin A$. But there exists no $A$-homomorphism $A^{\prime} \rightarrow A$, for it's easy to check that for any subring $B, \Omega^{(I)} \subset B \subset \Omega^{I}$, any $\Omega^{(I)}$ homomorphism $B \rightarrow \Omega^{I}$ is an injection. This example also has the property that for every prime ideal $\beta$ of $A, A / \beta$ is totally integrally closed, for, clearly, every unitary $f \in A / P[X]$ has a root in $A / P$. But since $A / \odot$ is an integral domain, this is easily seen to imply $A / \odot$ is in tegrally closed in the algebraic closure of its field of fractions (in fact it can be shown that $A / \mathbb{Q} \cong \Omega$ for every prime ideal $\mathbb{P}$ of $A$ ). Hence $A / \odot$ being totally integrally closed for every prime ideal $\odot$ of a ring $A$ doesn't imply $A$ is totally integrally closed.

REMARK 2. If $A$ is reduced and $f \in A[X]$ is unitary, it's easy to check that there is a "splitting ring" of $f$ unique up to $A$-isomorphism, i.e. a tight integral extension $B$ of $A$ with $B=A[S]$ where $S$ is the set of roots of $f$ in $B$ and such that if $C$ is any extension of $A$ with $C$ $=A[T]$ where $T$ is the set of roots of $f$ in $A$, there is an $A$-homomorphism $C \rightarrow B$.

REMARK 3. If $K$ is a field, any totally integrally closed extension $D$ of $K$ which is also a tight extension is just an algebraic closure of $K$.

REMARK 4. A tight extension need not be an integral extension, for if $K$ is a subfield of a field $E, E[[X]]$ is a tight extension of $K[[X]]$; but if $E$ is not algebraic over $K$, it's not an integral extension.

It's an open question whether $A$ totally integrally closed implies $S^{-1} A$ totally integrally closed for every multiplicative set $S \subset A$.

\section{BIBLIOGRAPHY}

1. M. Nagata, Local rings, Interscience, New York, 1962.

2. B. Eckmann and A. Schopf, Über injektive Moduln, Arch. Math. 4 (1953), $75-78$.

University of South Carolina and

UNIVERSITY OF KeNTUCKY 